\title{
Onset of natural convection in gas-gas system induced by bottom-up transient mass diffusion
}

\begin{abstract}
The onset of convection induced by transient mass diffusion in a stationary gas was succesfully predicted with transient instability theory and simulated using a computational fluid dynamics (CFD) scheme. 2D time-dependent simulations were conducted for bottom-up diffusion of a light gas in a stagnant heavy gas. The results of simulations were used to calculate the transient Rayleigh number adopted from the theory of Tan and Thorpe (1996 and 1999). The average transient maximum Rayleigh number from simulations is 707, which is close to the theoretical value of 817 for analogous bottom heating with constant heat flux. The simulated critical times of the onset of convection were in reasonably good agreement with the predicted values from theory.
\end{abstract}

Keyword: Gas-gas diffusion; Onset convection; Transient Rayleigh number 